\title{
Erratum to: Gold nanoflowers for 3D volumetric molecular imaging of tumors by photoacoustic tomography
}

Yuanyuan Jiang ${ }^{1, \S}$, Zijian Deng ${ }^{2, \S}$, Dan Yang ${ }^{3}$, Xin Deng ${ }^{1}$, Qi Li ${ }^{1}$, Yinlin Sha ${ }^{3}(\varangle)$, Changhui Li ${ }^{2}(\varangle)$, and Dongsheng $X u^{1}(\bowtie)$

'Beijing National Laboratory for Molecular Sciences, State Key Laboratory for Structural Chemistry of Unstable and Stable Species, College of Chemistry and Molecular Engineering, Peking University, Beijing 100871, China

2 Department of Biomedical Engineering, College of Engineering, Peking University, Beijing 100871, China

${ }^{3}$ Single-molecule and Nanobiology Laboratory, Department of Biophysics, School of Basic Medical Sciences, and Biomed-X Center, Peking University, Beijing 100191, China

${ }^{\S}$ These authors contributed equally to this work.

(C) Tsinghua University Press and Springer-Verlag Berlin Heidelberg 2017

\section{Erratum to}

Nano Research 2015, 8(7): 2152-2161

DOI 10.1007/s12274-014-0688-4

The original version of this article unfortunately missed acknowledgements part. The acknowledgements are as below.

\section{Acknowledgements}

We thank Prof. Decheng Wu for helping chemical synthesis. This work was supported by National Natural Science Foundation of China (Nos. 51121091, 21133001, 81421004, 21227803, and 21303004), Ministry of Science and Technology of China (Nos. 2014CB239303, 2013CB932601 and 2011CB808702), and the National Basic Research Program of China (973 Program, No. 2011CB707502). 